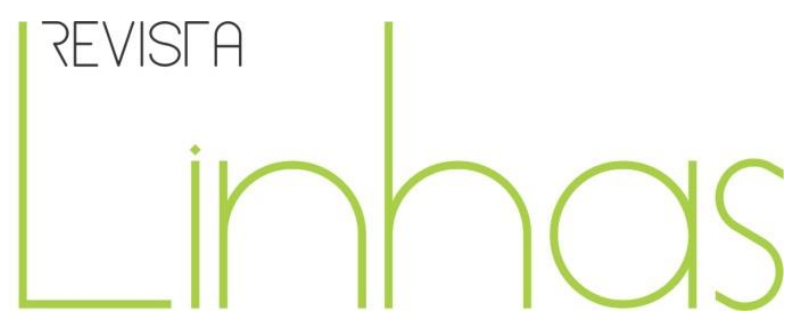

\title{
Redes pedagógicas y colectivos docentes conformados en torno de la documentación narrativa de experiencias pedagógicas
}

\begin{abstract}
Resumen
En las últimas décadas se han ido conformando en América Latina colectivos y redes de educadores que llevan adelante procesos autorregulados de formación, investigación educativa e innovación de las prácticas y discursos pedagógicos. Entre estas experiencias, abordaremos las que llevan adelante procesos de indagación narrativa y autobiográfica de sus prácticas y del mundo escolar informados en el dispositivo de documentación narrativa de experiencias pedagógicas. El artículo presenta algunos interrogantes acerca de los procesos de producción, validación, puesta en circulación y recepción del saber pedagógico desarrollados en el marco de colectivos y redes de docentes autores y lectores de documentos narrativos y sobre las modalidades de formación de docentes que tienen lugar en dichos procesos, en tanto espacios coparticipativos entre diversos actores educativos. A su vez, nos proponemos indagar los debates teórico-metodológicos que se plantean al interior de estas redes y colectivos docentes, que vienen a tensionar o interpelar al campo educativo en relación con la producción de saber pedagógico y la formación docente. Para ello, tomaremos aquí un caso particular, el del Programa de Extensión Universitaria "Red de Formación Docente y Narrativas Pedagógicas" de la Facultad de Filosofía y Letras de la Universidad de Buenos Aires, en Argentina. Al mismo tiempo, en el artículo reflexionamos respecto de la investigación (biográfica y narrativa) en educación como vía para la formación de los docentes y estrategia para la reconstrucción del saber pedagógico. También, sobre las posibilidades y desafíos de trabajar con "la/s voz/ces" de los docentes, reconstruirlas y reinterpretarlas, respetando sus propios puntos de vista, sus saberes y experiencias, su propio lenguaje, en marcos de producción colaborativos con los investigadores.
\end{abstract}

Palabras-clave: Investigación narrativa; Formación docente; Redes pedagógicas; Relatos pedagógicos.

\section{Para citar este artigo:}

DÁVILA, Paula Valeria; ARGNANI, Agustina. Redes pedagógicas y colectivos docentes conformados en torno de la documentación narrativa de experiencias pedagógicas. Revista Linhas. Florianópolis, v. 16, n. 32, p. $72-92$, set./dez. 2015.

DOI: $10.5965 / 1984723816322015072$

http://dx.doi.org/10.5965/1984723816322015072 


\section{Pedagogic networks shaped around the narrative documentation of pedagogic experiences}

\begin{abstract}
In recent decades in Latin America groups and networks of educators have been forming in self-regulating processes of training, educational research and innovation of educational discourses and practices. Among these experiences, we will describe those that carry out processes of autobiographical and narrative investigation through narrative documentation pedagogical experiences. The article presents some questions about the processes of production, validation, circulation and reception of pedagogical knowledge developed in the framework of networks of teachers authors and readers of narrative documents and on the modalities of teacher training that take place in these processes. At the same time, we intend to investigate the methodological debates that arise inside of these networks, coming to stress or question the educational field in relation to the production of pedagogical knowledge and teacher training. So, here take a particular case, the program "Network of training teaching and narratives teaching" from the Buenos Aires University, in Argentina. At the same time, the article reflects on strategy for the reconstruction of the pedagogical knowledge and research (biographical and narrative) in education as a means for teacher training. Also, about the possibilities and challenges of working with "the / s voice/ces" of teachers, rebuild them and reinterpreting them, respecting their own points of view, their knowledge and experience, their own language, in collaborative production frames with the researchers.
\end{abstract}

Keywords: Narrative research; Teacher training; Educational networks; Teaching stories.

\section{Redes pedagógicas e coletivos docentes criados em torno da documentação narrativa de experiências pedagógicas}

\section{Resumo}

Nas últimas décadas, foram-se organizando e ganhando forma na América Latina coletivos e redes de educadores que levam adiante processos autorregulados de formação, investigação educativa e inovação das práticas e discursos pedagógicos. Entre essas experiências, abordaremos as que desenvolvem processos de investigação narrativa e autobiográfica de suas práticas e do mundo escolar informados no dispositivo de documentação narrativa de experiências pedagógicas. $O$ artigo apresenta alguns questionamentos sobre os processos de produção, validação, circulação e recepção do saber pedagógico desenvolvidos no contexto de coletivos e redes de docentes e autores e leitores de documentos narrativos e sobre as modalidades de formação de docentes que acontecem nesses processos, enquanto espaços coparticipativos entre diversos atores educativos. Por nossa vez, propomo-nos indagar os debates teóricometodológicos que se inserem no interior dessas redes e coletivos docentes, que vêm tensionar ou interpelar o campo educativo em relação com a produção de saber pedagógico e a formação docente. Para isso, tomaremos aqui um caso particular, o do Programa de Extensión Universitaria "Red de Formación Docente y Narrativas Pedagógicas" da Faculdade de Filosofia e Letras da Universidade de Buenos Aires, na Argentina. Ao mesmo tempo, no artigo refletimos acerca da investigação (biográfica e narrativa) em educação como via para a formação dos docentes e estratégia para a reconstrução do saber pedagógico. Também, sobre as possibilidades e desafios de trabalhar com "a(s) voz(es)" dos docentes, reconstruí-las e reinterpretá-las, respeitando seus próprios pontos de vista, seus saberes e experiências, sua própria linguagem, em contextos de produção colaborativos com os investigadores.

Palavras-chave: Investigação narrativa; Formação docente; Redes pedagógicas; Relatos pedagógicos. 
Según indican diversos estudios previos en los que viene trabajando este equipo de investigación ${ }^{1}$, resulta limitada la disponibilidad de canales y espacios para la formación horizontal entre docentes que sean pensados en torno de las propias experiencias pedagógicas. Aunque de manera menos explícita y masiva, actualmente continúan actuando e implementándose dispositivos de administración y gestión escolares centralizados y verticalistas, orientados por la racionalidad del control técnico y fundamentados en la preeminencia del saber experto, especializado y científico-técnico que han habilitado posiciones diferenciadas y jerarquizadas respecto a la capacidad de los distintos actores del campo pedagógico para producir saberes legítimos. Dichas políticas han contribuido al confinamiento de los docentes en posiciones subordinadas y descalificadas, así como a la desacreditación de sus saberes, discursos y prácticas pedagógicas. Esto se ha traducido, además, en la limitación de espacios de formación y de investigación en los cuales los docentes puedan participar e intervenir pedagógicamente para la construcción de discursos y saberes del campo educativo (Suárez, 2009, 2007).

Asimismo, en diversos trabajos hemos identificado las variadas y múltiples dificultades vinculadas a la disponibilidad y habilitación de espacios y tiempos para el trabajo colaborativo entre diversos actores del sistema educativo. En este sentido, resulta limitada la articulación entre la investigación educativa desarrollada desde ámbitos académicos y las experiencias escolares. Se identifican "problemas de comunicación" entre el mundo de la investigación académica y el mundo de las prácticas pedagógicas y entre sus habitantes, es decir, investigadores y docentes. Maestros y profesores no ven en la investigación científica ni una lente para mirar, interpretar y recrear sus prácticas profesionales, ni un espacio de conversación e interpelación, ni la solución a los múltiples problemas prácticos con los que se enfrentan día a día.

\footnotetext{
${ }^{1}$ Proyectos de Investigación "La construcción social del cambio educativo. desigualdades y diferencias en el campo pedagógico" (UBACyT 2011-2014) dirigido por el Dr. Daniel Suárez; “La construcción social del cambio educativo. Una mirada político pedagógica a las continuidades y rupturas en las formas de lo escolar" (UBACyT 2008-2010 F028), dirigido por el Dr. Daniel H. Suárez y la Lic. Dora González; “Pedagogos, docentes y relatos de experiencia. Saberes pedagógicos y experiencias formativas producidas en la trayectoria profesional" (UBACyT 2008-2010 F096), dirigido por la Dra. Andrea Alliaud y el Dr. Daniel H. Suárez. Instituto de Investigaciones en Ciencias de la Educación. Facultad de Filosofía y Letras. Universidad de Buenos Aires.
} 
Frente a estas políticas y discursos hegemónicos vinculados a la producción del conocimiento educativo, diversos movimientos de docentes sostienen experiencias alternativas de investigación, formación y producción de saber pedagógico. Se trata de procesos de organización y conformación de colectivos y redes de maestros en distintos lugares de la región ${ }^{2}$ vinculados con la reconstrucción del saber pedagógico, la activación de la memoria de la escuela y la movilización e intervención en el campo educativo 3 .

Como parte de una red plural y teñida de los colores que aportan las diferentes culturas y experiencias latinoamericanas, también en Argentina se han desarrollado distintas formas de organización y de trabajo pedagógico entre educadores, que apuntan a diseñar y poner en práctica líneas de acción diversas y articuladas, como así también a estrechar vínculos de trabajo colaborativo con otras instituciones y organizaciones ${ }^{4}$. Estas redes y colectivos sostienen como uno de sus propósitos centrales la posibilidad de

\footnotetext{
${ }^{2}$ Como antecedentes importantes e influyentes en el campo pedagógico latinoamericano, y entre las múltiples experiencias de la región, vale destacar el Movimiento Pedagógico Nacional y la Expedición Pedagógica Nacional de Colombia; los Centros de Autoformación Docente en Perú; y la Red Iberoamericana de Colectivos Escolares y Redes de Maestros que hacen Investigación desde su Escuela, actualmente conformada por: BRASIL: Red RIE (Red de Investigación en la Escuela) - COLOMBIA: Red CEE (Red de Cualificación de Educadores en Ejercicio); Movimiento Expedición Pedagógica; REDLENGUAJE (Red Latinoamericana para la Transformación de la Formación Docente en Lenguaje. Red de Lenguaje); Red ESMAESTRO (Escuela del Maestro, Medellín) - ESPAÑA: Red IRES (Investigación y Renovación Escolar) MÉXICO: REDIEEM (Red de Investigadores/as Educativos/as en el Estado de México); Red RETE (Red Estatal para la Transformación Educativa en Michoacán); Red de Educadores que hacen Gestión Compartida; REDLENGUAJE (Red Latinoamericana para la Transformación de la Formación Docente en Lenguaje); Red LEE (Red de Lenguajes por la Transformación de la Escuela y la Comunidad) - PERÚ: CPDHIEC (Colectivo Peruano de Docentes que Hacen Investigación e Innovación desde su Escuela y Comunidad) - VENEZUELA: Red CIRES (Colectivos de Investigación y Redes Escolares) - URUGUAY: Red DHIE Uruguay (Centro Regional de Profesores del Litoral, DFyPD, ANEP, Salto) - ARGENTINA: Colectivo Argentino de Educadoras y Educadores que hacen Investigación desde la Escuela; Red DHIE (Red de Docentes que Hacen Investigación Educativa); Red IPARC (Red Investigación Participativa Aplicada a la Renovación Curricular); Red DRIA (Red de Docentes que Realizan Investigación desde el Aula); Red ORES (Red de Orientadores Escolares); Grupo de investigación IFIPRACD (Investigación en Formación Inicial y Práctica Docente); Red de Formación Docente y Narrativas Pedagógicas (FFyL-UBA); REDISP (Red de Institutos Superiores de Profesorado de Gestión Pública Estatal de Santa Fe); REDINE (Red de Investigación Educativa- Universidad Nacional de Misiones); AGCEJ (Asociación de Graduados Ciencias de la Educación de Jujuy); Unidad de Investigación "Educación, Actores Sociales y Contexto Regional" (F. H. y Cs. Soc. - UNJu).

${ }^{3}$ Pilar Unda Bernal plantea una breve caracterización de las redes pedagógicas, a las que entiende como: a) producto de la insatisfacción y la crítica a las prácticas existentes de formación; b) alternativas frente a la incapacidad de los modelos de investigación diseñados por investigadores externos a la escuela para comprometer acciones y solucionar los problemas educativos de los centros escolares; c) estructuradas sobre la necesidad de consolidar concepciones y prácticas diferentes a las de la cultura tradicional hegemónica en la escuela. Además, Unda Bernal aclara que "en todas ellas se ponen en juego otras maneras de concebir al maestro" (Unda Bernal y Martínez Boom, 2002: 5).

${ }^{4}$ Podemos señalar, entre otras experiencias, al Colectivo Argentino de Docentes que Hacen Investigación desde la Escuela, que reúne a un conjunto de educadores y educadoras que se desempeñan en organizaciones sindicales, instituciones del sistema educativo formal y en experiencias pedagógicas alternativas. Ver Colectivo Argentino de Docentes que Hacen Investigación desde la Escuela, 2009.
} 
establecer relaciones de cooperación entre docentes e investigadores, en pos de la reconstrucción del saber pedagógico desde las voces y experiencias de los propios protagonistas y la movilización político-pedagógica en el campo educativo. Entre estas experiencias podemos identificar a las redes y colectivos docentes que llevan adelante procesos de indagación narrativa y autobiográfica de sus prácticas y del mundo escolar informados en el dispositivo de documentación narrativa de experiencias pedagógicas.

Nos proponemos en este trabajo dar a conocer y problematizar una experiencia que venimos desarrollando en el marco de un Programa de Extensión Universitaria de la Facultad de Filosofía y Letras, denominado "Red de Formación Docente y Narrativas Pedagógicas", en curso desde el año 2010. Esta Red articula colectivos de docentes e investigadores que, desde diversos espacios geográficos, organizacionales e institucionales ${ }^{5}$, desarrollan procesos horizontales de formación basados en la reconstrucción narrativa y reflexiva de la experiencia educativa y de los saberes pedagógicos construidos en torno de ella, su sistematización, publicación, difusión y deliberación públicas, informados en el dispositivo de documentación narrativa de experiencias pedagógicas, que se describirá más adelante.

Consideramos que esta experiencia de trabajo en red encuentra un ámbito con fuertes potencialidades en el marco de la extensión universitaria, ya que plantea una perspectiva integral de las prácticas de extensión, investigación y docencia en vistas a desarrollar "nuevas forma de enseñar, aprender e investigar" en contacto más directo con los actores educativos, sus problemas, demandas y necesidades.

\footnotetext{
${ }^{5}$ En la Red participan como nodos: dos institutos de formación docente públicos estatales de la Provincia de Buenos Aires ( $\mathrm{N}^{\circ} 100$ y 103), un instituto de formación docente de gestión social de la Ciudad de Buenos Aires (Instituto Alfredo Palacios), la Subsecretaria de Igualdad de Género y Tercera Edad del Municipio de Quilmes, la Dirección de Políticas Ambientales del Municipio de Morón, la Jefatura Regional Educativa $\mathrm{N}^{\circ} 3$, La Matanza, de la Provincia de Buenos Aires, el sindicato docente AMSAFE, el Grupo "Memoria Docente y Documentación Pedagógica" - Facultad de Filosofía y Letras (UBA).
} 


\begin{abstract}
"Aprendemos a tender redes, tramas y tejidos con otras instituciones y otros actores para resignificar espacios, como un modo de crear y recrear metodologías de trabajo, de generar o fortalecer procesos de organización y participación para hacer realidad sueños, utopías." (Red DHIE, 2009: 56)
\end{abstract}

En el Programa desarrollamos e implementamos un dispositivo de trabajo que genera relaciones horizontales entre docentes e investigadores y se orienta a la producción de relatos de experiencias escritos por los propios docentes, pretendiendo poner en foco la reconstrucción e interpretación de las experiencias y saberes pedagógicos que los tienen como protagonistas. Desde una perspectiva particular de investigación-formación-acción, nos proponemos establecer un espacio de coinvestigación, formación y trabajo conjunto entre colectivos de docentes narradores, redes educativas, investigadores, docentes y estudiantes universitarios en virtud de la reconstrucción narrativa y reflexiva de la experiencia escolar y de los saberes pedagógicos construidos en torno de ella.

El dispositivo de documentación narrativa de experiencias pedagógicas, supone la indagación de las propias experiencias y prácticas educativas, a través de su narración, sistematización, comunicación y debate. Los educadores llevan adelante procesos de formación horizontal entre colegas, mediante la escritura, la lectura, la conversación, la publicación y la circulación de sus relatos de experiencia. Esto constituye una estrategia de formación, en la medida en que permite resignificar y reorientar las prácticas de manera sistemática, reflexiva y crítica. Pero al mismo tiempo, este proceso supone la reconstrucción, disposición pública y deliberación colectiva en torno del saber pedagógico puesto en juego en las prácticas de los docentes. Para ello, el dispositivo de trabajo se orienta a la producción individual y colectiva de relatos pedagógicos que den cuenta de los modos en que los docentes estructuran sus vidas profesionales, dan sentido a sus prácticas educativas y se presentan a sí mismos como activos conocedores del mundo escolar, a través de la indagación de las propias experiencias y prácticas educativas, su narración, sistematización, comunicación y debate (Suárez, 2009; Suárez, Ochoa y Dávila, 
2005). Cuando narran experiencias pedagógicas que los tienen como protagonistas, los docentes reconstruyen interpretativamente sus trayectorias profesionales y les otorgan sentidos particulares a sus experiencias. En el mismo movimiento, los docentes reelaboran reflexivamente esas experiencias y se reposicionan respecto de ellas, ya más distanciados que cuando las vivieron (Suárez, 2011).

Una vez que estos relatos circulan y se vuelven públicamente disponibles, esas historias de enseñanza se constituyen en documentos pedagógicos. En este proceso formativo y de investigación por la acción, los educadores se tornan autores de materiales documentales que dan cuenta de experiencias y saberes pedagógicos que los tienen como protagonistas y los interpelan como habitantes del mundo escolar.

Uno de los propósitos del dispositivo es generar un espacio de trabajo pedagógico colectivo entre educadores (docentes e investigadores) que promueva y habilite la constitución de una comunidad de docentes narradores de experiencias pedagógicas. El trabajo al interior de los colectivos supone la reconstrucción y deliberación colectiva respecto al saber pedagógico puesto en juego en las prácticas de los docentes y su disposición pública para la reflexión, la conversación informada, la interpretación y el intercambio, entre investigadores y docentes narradores, en torno a los documentos pedagógico producidos por los docentes (Suárez, Dávila y Ochoa, 2008, 2009), con la intención de constituir “comunidades de atención mutua” (Connelly y Clandinin, 1995). Es decir, se pretende generar un espacio de trabajo co-participado que promueva la constitución de colectivos de docentes narradores ${ }^{6}$, entendidos como comunidades pedagógicas, donde se generan y proyectan relaciones horizontales y espacios cooperativos.

En los colectivos "enredados" tienden a confluir voluntades e intereses específicos en función de la definición de objetivos comunes; se asumen compromisos colectivos para la reflexión e investigación; se reconocen las responsabilidades compartidas y se abrazan las diferencias, ya sean teóricas, metodológicas, pedagógicas y/o políticas. Al

\footnotetext{
${ }^{6}$ Denominamos de este modo al conjunto de docentes que participa de instancias de escritura, lectura, intercambios y reflexión pedagógica, en el maro del dispositivo de documentación narrativa. La cantidad de integrantes, las formas de coordinación de los mismos, y las modalidades de encuentro y trabajo varían en función de las definiciones de cada colectivo -y del Nodo al que pertenecen, en caso de formar parte de la Red en cuestión. Consideramos que dichos colectivos pueden ser pensados como formas organizativas de investigación, formación e intervención en el campo de la pedagogía pública.
} 
favorecer estructuras más flexibles y vínculos de colaboración, el trabajo en red permite extender y diversificar actividades, experimentar nuevos desarrollos, fortaleciendo el trabajo de cada uno de los nodos y generando a la par nuevos espacios y producciones, como consecuencia de la sinergia y el intercambio producido.

Las redes y colectivos promueven la recreación de las modalidades convencionales de organización colectiva de los docentes (sistémicas, burocráticas, centralizadas, jerárquicas, corporativas, disciplinadas) y de organización social, para la producción, circulación, evaluación, validación y uso del saber pedagógico (Suárez y Argnani, 2011). Se ensayan, exploran y redefinen otras estructuras de participación y toma de decisiones y se revisan las relaciones de saber y poder en esas comunidades de prácticas y discursos entendidas como “comunidades de atención mutua" (Connelly y Clandinin, 1995). Asimismo, las prácticas organizativas, requisitos para la movilización y la acción colectiva, constituyen también "resultados" de la formación. Es decir, se expresa el carácter formativo del movimiento y la organización colectiva. Al involucrarse y ser parte de las redes se fortalecen, amplían y revisan las instituciones, organizaciones o colectivos, planteando nuevas articulaciones y relaciones y otras formas de organización pedagógica en las cuales los maestros son protagonistas. Por otro lado, el trabajo en red favorece diversas formas y vías de encuentro y participación, ya sean presenciales o virtuales (de lectura entre pares, reuniones de coordinación, publicaciones colectivas, intercambio de experiencias o desarrollo de actividades conjuntas) que permiten la circulación y la producción colectiva del saber pedagógico.

Al desarrollar un escenario donde la articulación se entiende como una integración activa, el trabajo en red propicia el reconocimiento de la dimensión social y comunitaria de los problemas y habilita la posibilidad de pensar juntos los procesos necesarios para la transformación, potenciando a su vez la posibilidad de intervención. Es decir, permite ampliar los horizontes de acción así como fortalecer los vínculos entre los educadores, docentes e investigadores para configurarse como “(...) una alternativa viable para la producción de conocimientos pertinentes a la realidad educativa que se aspira transformar" (Cardelli, 2009: 11).

El "entramado de hilos y nudos" permite a la red asumir la "vitalidad del intercambio y la inmensa perspectiva creadora del aprender con otros" (Awad, 2002:121). 
Es en el tejido de la red donde los docentes se encuentran, comparten y conversan, "realizan producciones e intercambios de conocimientos acerca de sus propias prácticas y contextos socioeducativos" transformándose en "sujetos de experiencia” y en "sujetos de saberes", construyendo formas propias y singulares de ser maestros y de hacer escuela (Duhalde, M. y otros, 2009: 22-23).

En este sentido, se tensiona y redefine la identidad docente al posicionarse como productor y portador de saberes pedagógicos, en un proceso colectivo de producción de conocimientos y de desarrollo de otros modos de organización, orientados a la democratización de la escuela. También, el papel de los investigadores se juega desde una posición peculiarmente diferente de la "tradicional”, en una relación distinta de la acostumbrada: de co-participación, cooperación y reciprocidad, ya que sostenemos que es desde un lugar de simetría con los docentes narradores y en el marco de esta relación de horizontalidad donde se puede alojar la promoción de otro modo de conocer e investigar en pedagogía.

Uno de los puntos nodales del dispositivo de documentación narrativa de experiencias pedagógicas es la posición cualitativamente distinta de la habitual que procura impulsar para los docentes: como actores centrales de la producción de conocimiento pedagógico y como autores protagonistas en la tarea de contar sus experiencias pedagógicas. Pero también interesa profundizar en esta nueva posición en que los investigadores son puestos a jugar. Una posición peculiarmente diferente de la tradicional, en una relación distinta de la acostumbrada: de co-participación, cooperación y reciprocidad. Es desde este lugar de simetría con los docentes narradores, y en el marco de esta relación de horizontalidad, donde podría alojarse la promoción de otro modo de conocer e investigar en pedagogía.

Este reposicionamiento entre investigadores y docentes, que se da a la hora de trabajar conjuntamente en la elaboración, edición pedagógica y publicación de los relatos, colabora a traccionar el desplazamiento de las concepciones de formación y desarrollo profesional docente desde un modelo centrado en el "conocimiento experto" hacia un enfoque que valoriza la práctica profesional y el saber de la experiencia como fuentes de conocimiento, aprendizaje, indagación y construcción de estrategias pedagógicas, y que considera también a éste como "saber experto". Es en este sentido 
que nos referimos a la necesidad de un reposicionamiento de saberes, conocimientos y sujetos en el campo pedagógico, pues la documentación narrativa de experiencias es una estrategia de trabajo pedagógico entre docentes e investigadores que supone prácticas de investigación-acción colaborativas y participantes, que otorgan un valor fundamental a los procesos de autocomprensión y comprensión mutua como forma de producir cambios (Anderson y Herr, 2007).

El Programa “Red de Formación Docente y Narrativas Pedagógicas” retoma estos sentidos al constituirse como una iniciativa de intervención, formación e investigación pedagógicas orientada a involucrar de manera activa a los docentes en la producción y reconstrucción crítica de sus saberes pedagógicos, y permite la disposición pública de un corpus de relatos pedagógicos que interpelan y tensionan los discursos y la teoría educativa pública. De este modo, la Red se proyecta hacia la transformación democrática de los discursos y prácticas pedagógicas, como una forma de organización social para la producción del saber pedagógico, en una comunidad interpretativa y de atención mutua, para la intervención político-pedagógica en el campo educativo.

\section{Una red en torno a los relatos de experiencia}

A través de la Red se articulan colectivos de educadores con espacios dedicados a la formación (Institutos Superiores de Formación Docente y la Facultad de Filosofía y Letras - UBA, asociaciones sindicales docentes) y actores de comunidades locales (grupos de educadores que participan a través de Programas, Direcciones y Secretarías municipales), potenciando de esta manera la transferencia e intercambio de saberes pedagógicos, con vistas a fortalecer los procesos formativos de los actores individuales y colectivos involucrados.

Como miembros de la Red, los Nodos participan de los espacios y actividades que se desarrollan de forma conjunta y, por su parte, llevan adelante acciones de manera autónoma y en articulación con actores locales, vinculadas con las temáticas específicas que cada Nodo aborda. Una de las actividades centrales que es común a todos los Nodos tiene que ver con el despliegue de procesos de documentación narrativa, de experiencias pedagógicas a través de los cuales los colectivos de docentes indagan narrativamente sus 
propias experiencias pedagógicas. Esto ha configurado un proceso particular de documentación narrativa que ha permitido sostener de manera paralela procesos de indagación, registro y reflexión en torno de experiencias pedagógicas de docentes en diversos colectivos anclados en los diferentes nodos, y procesos de documentación pedagógica de las experiencias formativas y de acompañamiento de los coordinadores de dichos colectivos ${ }^{7}$. Esto permite pensar la formación docente como un trabajo en red, marcado por la confluencia de perspectivas, trayectorias y contextos institucionales y habilita la reflexión sobre las condiciones de producción de saber pedagógico que se configuran en cada uno de los escenarios institucionales: qué sucede en los profesorados, en las instancias municipales de formación, en los sindicatos y en las universidades que participan de la Red. Este proceso nos ha conducido a re-pensar la formación (tanto la formación docente como la formación en documentación narrativa) al poner en tensión las implicancias y modalidades de organización que asume el trabajo docente y las posiciones de sujeto que los educadores asumen en al campo pedagógico respecto de la producción, validación y circulación de conocimiento.

Desde la Red desarrollamos, además, Ateneos de lectura, interpretación y debate pedagógicos de los documentos narrativos producidos por los docentes en el marco de la Red. La dinámica de estos encuentros, en general, consisten en una ronda de lectura en plenario de relatos seleccionados (tres o cuatro relatos) y posteriormente la apertura de una instancia de comentarios, preguntas a los autores e intercambios con los asistentes. Resulta central dimensionar la relevancia de estos encuentros en términos de reconocimiento, horizontalidad y voces en juego. No sólo por la lectura y reflexión en torno de los documentos pedagógicos narrativos escritos por docentes, sino porque estos espacios se fueron consolidando también como instancias de indagación y formación: en el marco de los debates de los Ateneos se fueron abordando, a partir de la reflexión en torno de los relatos, inquietudes vinculadas a la posición y la identidad docente frente a la producción de conocimientos en el mundo escolar; a los criterios para

\footnotetext{
7 En el marco del "Seminario-taller de formación para coordinadores de procesos de documentación narrativa de experiencias pedagógicas" se realizaron encuentros de formación con los coordinadores que estaban a cargo de acompañar los procesos de documentación narrativa de los colectivos docentes en cada Nodo; así como también participaron docentes y estudiantes interesados en incorporarse al trabajo de la Red e interiorizarse sobre la documentación narrativa de experiencias pedagógicas y la investigación (auto)biográfica y narrativa. Esta propuesta formativa se estableció a partir de 2011 como un curso de extensión la Secretaría de Extensión Universitaria de la Facultad de Filososfía y Letras, UBA.
} 
validar lo producido desde estos espacios y formas de organización; a las relaciones y tensiones con las administraciones públicas y la academia; a las formas no convencionales de trabajo y formación en espacios horizontales.

Por otro lado, en el intento de generar conversaciones y, a su vez, promover instancias de articulación con otras redes de docentes e investigadores del país y de América Latina la Red desarrolló encuentros públicos y semipúblicos de intercambio y debate entre diversos actores del campo educativo, que se articularon con una serie de seminarios, talleres y actividades a los que hemos denominado Seminarios Itinerantes ${ }^{8}$. En este marco se ofrecieron espacios de encuentro e intercambio en los que docentes, investigadores, estudiantes universitarios, referentes de otras redes, especialistas y educadores ${ }^{9}$ conversaron en torno a diversas experiencias de formación e investigación que tienen lugar desde formas de organización colectiva y de trabajo pedagógico entre educadores. Se desarrollaron también Ateneos de docentes narradores ${ }^{10}$ con la participación de investigadores o referentes de otras redes que intervinieron como comentaristas invitados; talleres de documentación narrativa de experiencias pedagógicas; actividades abiertas ${ }^{11}$; visitas y conversatorios con integrantes de diversas redes. Todas estas actividades se desarrollaron de manera articulada, como un itinerario o un recorrido por los distintos nodos de la Red. La idea era transitar y conocer los territorios, las instituciones, y los integrantes (coordinadores y docentes) de cada Nodo.

\footnotetext{
${ }^{8}$ Se trata del Seminario Itinerante "Narrativas, Formación Docente e Investigación Educativa" en el año 2011 y el Seminario Internacional Itinerante "Formación, narrativas y redes pedagógicas" en el año 2012. Documentos e información disponible en: <http://www.memoriapedagogica.com.ar/red/seminario_itinerante.html>

${ }^{9}$ Participaron representantes de la Red NAUE; de la Red LEO y de la Universidad Nacional Autónoma (México); de la Universidad de Antioquia y la Casa del Maestro (Colombia); del Colectivo de Docentes Autores (Universidad de Chile); de la Universidad del Estado de Bahía y de la Universidad Federal de Rio Grande do Norte (Brasil); y de la Universidad de Paris 13 Nord (Francia).

${ }^{10}$ Nos referimos a los Ateneos de Docentes Narradores y Conversatorios "Narrativas, autobiografías y formación", desarrollado el 26 de octubre de 2011 en la Casa de la Memoria y la vida, coordinado por el Nodo Morón, e "Historias de vida, relatos de experiencias y formación" desarrollado el 27 de octubre en el Instituto de Formación Docente $\mathrm{N}^{\circ} 100$, Nodo Avellaneda. En la edición 2012 del Seminario Itinerante se desarrolló un Ateneo de Docentes Narradores coordinado por el Nodo Quilmes, el 24 de abril en el Museo de Artes Visuales Víctor Roverano de la Ciudad de Quilmes; y el Ateneo y Conversatorio "El saber de la experiencia: Relatos autobiográficos, formación y redes pedagógicas", desarrollado en el Nodo Filosofía y Letras de la Red, el día 26 de abril.

${ }^{11}$ En el marco del Seminario Itinerante del año 2011 se llevó a cabo en la Facultad de Filosofía y Letras/UBA una Mesa Redonda en la que participaron investigadores dedicados a la formación de docentes y la investigación autobiográfica y narrativa. La Mesa contó con los aportes de Andrea Alliaud - Universidad de Buenos Aires - OEl/ Argentina; Christine Delory-Momberger - Universidad de París 13 Nord/ Francia; Elizeu Clementino de Souza - Universidad del Estado de Bahía/ Brasil y Maria da Conceição Passeggi - Universidad Federal de Rio Grande do Norte/ Brasil.
} 
De esta manera, a través de las múltiples vinculaciones y articulaciones con actores diversos del campo pedagógico, el Seminario Itinerante intentó contribuir a la movilización político-pedagógica de los docentes y promover la organización colectiva para el debate en educación.

Como señalábamos, desde la Red participamos, a su vez, de las acciones desplegadas por otras redes de docentes investigadores del país y de América Latina, entre ellas vale destacar la Red Iberoamericana de Colectivos Escolares y Redes de Maestros que hacen investigación desde su escuela ${ }^{12}$ y la Red Latinoamericana de Narrativas, Autobiografías y Educación (Red NAUE) ${ }^{13}$, y del mundo, como la Red BioGrafia / Red científica de investigación biográfica en educación América latina-Europa ${ }^{14}$. Esta apuesta de construir "redes de conocimientos" con otros colectivos se desprende de la intención y la necesidad de intercambiar y aprender también de los aportes de campo pedagógico y académico.

Las acciones de publicación de la Red se centraron en la organización y generación de espacios de encuentro y conversación entre diversos actores del campo pedagógico que ejercieron a su vez como disparadores: se trató de espacios para el convite y la disposición de los relatos, y su posterior circulación y esparcimiento por diversos canales, la mayoría de las veces, de la mano de los participantes allí reunidos. Así, la publicación de

\footnotetext{
${ }^{12}$ La Red Iberoamericana persigue la construcción de un movimiento político- pedagógico capaz de incidir en decisiones de políticas públicas en materia de educación en cada país y en la región en su conjunto. Comparten este proyecto redes y organizaciones de Argentina, Brasil, Colombia, España, México, Perú, Uruguay y Venezuela. Ver: Universidad Pedagógica Nacional (2002), Memorias. III Encuentro Iberoamericano de Colectivos Escolares y Redes de Maestros que hacen Investigación desde su Escuela. Santa Marta, Colombia, 2002.

${ }^{13}$ La Red NAUE tiene como propósito establecer proyectos y líneas de trabajo de cooperación horizontal para la realización de investigaciones, publicaciones y actividades académicas vinculadas a estudios centrados en la indagación narrativa y (auto)biográfica en educación. La Red establece vínculos de cooperación y lazos institucionales entre: Universidad de Buenos Aires, Argentina; Universidade do Estado da Bahia y Universidade Federal do Rio Grande do Norte, Brasil; Universidad de Antioquia, Medellín, Colombia; Universidad de Chile y Universidad Pedagógica Nacional, México; entre otras instituciones universitarias de América Latina.

${ }^{14}$ La Red BioGrafia reúne investigadores de países de América latina y Europa, cuyos trabajos pertenecen al dominio de la investigación biográfica en educación y formación. La misma tiende a divulgar y promover las investigaciones en ese dominio, en ambos continentes, adentrándose en temas relacionados con la actualidad sociopolítica y económica de la educación. Los diferentes enfoques de realidades propias de la historia e inquietudes educativas de cada país permiten revelar puntos en común y específicos, habilitar perspectivas de trabajo mancomunado y someter a prueba en diversos campos, conceptos y cuestiones de la investigación biográfica.
} 
los relatos en el marco de la Red adquirió relevancia como evento, como instancia de encuentro, como ocasión de apertura de canales de circulación de los relatos, y como “excusa” de movilización de los docentes. Estos encuentros se fueron configurando como ámbitos de debate, no sólo acerca de los saberes y experiencias pedagógicas contenidos en los relatos sino que también se desarrollaron allí intercambios y reflexiones entre los participantes sobre los criterios de validación de los saberes pedagógicos producidos desde formas de organización colectivas y modalidades co-participadas, como son los colectivos y redes de docentes narradores.

Como consecuencia del trabajo de la Red, cada vez más docentes se han interesado en contar lo que (les) sucede con la enseñanza, en preguntarse y conversar sobre lo que hacen y piensan, en reflexionar e indagar sobre sus mundos escolares y lo que interpretan de ellos. $\mathrm{Y}$ junto con ese número creciente de docentes narradores e indagadores, el volumen y la densidad del corpus de relatos de experiencias pedagógicas también se fue multiplicando, diversificando, ampliando. $\mathrm{Y}$ también los espacios de encuentro, debate y discusión pedagógicas, donde las voces de los docentes y los relatos de experiencias fueron cobrando centralidad, al participar diversos actores de una conversación, interpretación y resignificación de las prácticas y saberes mediados por el relato.

A lo largo del trabajo desarrollado, hemos advertido que las formas que adopta el trabajo en red son muy variadas (radiales, anillos, mallas) y a su vez definen diversas modalidades de participación y toma de decisiones en los colectivos, problematizando y expendiendo la idea de red como forma de organización sin un centro, horizontal y democrática. La articulación de organizaciones y experiencias heterogéneas como nodos de una red permite poner de manifiesto la diversidad de prácticas pedagógicas y de formas de "hacer escuela" de acuerdo con los entornos culturales, los contextos geográficos e históricos. Advertimos que la articulación de organizaciones y experiencias diversas vehiculiza no sólo la posibilidad de profundizar el intercambio y ampliar perspectivas, sino también aproximarse a otras organizaciones pedagógicas para la movilización e intervención pública.

En este sentido, la Red de Formación Docente y Narrativas Pedagógicas acerca y pone en diálogo diferentes formas de saber y de nombrar el mundo y la experiencia 
escolares, con el propósito central de generar "ecologías de saberes" (Santos, 2006), promoviendo comunidades interpretativas descentradas, plurales, democráticas y orientadas a la transformación.

Por su parte, algunas dificultades encontradas hasta el momento están relacionadas con los tiempos y ritmos de cada una de las instituciones involucradas en dicha Red y sus proyectos, distintos de los que implican las demandas que conlleva una propuesta colectiva de investigación-formación-acción. Sin embargo, la invitación a organizar un espacio participativo, con la modalidad de red, en la que interactúan distintos tipos de organizaciones (municipios, institutos de formación docente, sindicatos, universidades) tensiona la idea de acompasar ritmos y tiempos, y desafía a pensar nuevos modos de articulación y de participación para la toma de decisiones conjuntas. La noción misma de "red" se encuentra en permanente discusión, y este debate y resignificación permanente acompaña el proceso de construcción de la propia identidad colectiva como organización (Duhalde y otros, 2009). Estas disputas en torno a los sentidos que asume la idea de red, compartido con otras redes y colectivos, nos permite dimensionar las potencialidades y también los límites de la construcción en red y las múltiples posiciones, negociaciones y tensiones que supone el trabajo co-participado y plural.

\section{Hacia formas co-participativas de producción de conocimientos}

Ahora bien, decíamos que la Red de Formación Docente y Narrativas Pedagógicas es una red "tejida", entrelazada por medio de relatos de experiencia, y que estos relatos son generados, producidos a lo largo de un itinerario y a través de un dispositivo específico, el de documentación narrativa de experiencias pedagógicas. En tanto modalidad particular de investigación-formación-acción, creemos que este dispositivo encuentra un ámbito con fuertes potencialidades en el marco de la extensión universitaria, con vistas a sostener lazos y articulaciones no sólo entre miembros de la comunidad académica, sino entre los investigadores y otros sujetos de conocimiento (actores sociales, movimientos y organizaciones) para la producción y validación del saber. Desde la Red sostenemos la conveniencia y la necesidad de trabajar con otros 
actores para la construcción de un conocimiento más sensible, sofisticado y potente que promueva relaciones de colaboración y espacios co-participativos entre los diversos actores como otro modo de debatir y discutir las distintas dimensiones que abarca el trabajo docente, para generar una posición como colectivo que le permita intervenir en el debate pedagógico público desde su propio hacer y saber, con su propia voz y con sus propias palabras.

Así, la configuración de las relaciones y jerarquías político-institucionales establecidas en el campo educativo que obturan la posibilidad de los docentes de participar en la producción sistemática y validada de saberes pedagógicos se ven cuestionadas por las “redes de conocimiento". La producción co-participativa de saberes trae aparejada una tensión en la política de conocimiento, así como el establecimiento de otro tipo de relaciones entre los distintos actores del campo educativo: los supuestos epistemológicos, las lógicas de producción, validación y circulación de los saberes y las posiciones asignadas a los sujetos se ven disputadas.

La generación de dispositivos específicos, elaborados colaborativa y horizontalmente, sin dudas contribuye a generar las condiciones de recepción para que opere ese reposicionamiento profundamente crítico de sujetos y saberes al que hacíamos referencia. No se trata aquí de determinar quién "autoriza” los saberes puesto en juego, ni con qué reglas deberían ser validados; se trata más bien, de la construcción de otras reglas de discurso y de prácticas donde el mismo concepto de “validación” es tensionado y redefinido (Suárez, 2009) 15 . Podemos señalar que las redes se constituyen como espacios de construcción de saberes y de sujetos en tanto configuran un entramado de experiencias, relaciones y sentidos en el cual los elementos heterogéneos, sujetos, instituciones y grupos redefinen su propia identidad desde la participación activa y la articulación con otros. La heterogeneidad, como elemento constitutivo de las redes y como política de re-conocimiento del otro, se enlaza en una trama de voces plurales, en una malla de nudos y nodos, de identidades en construcción, de procesos colectivos en

\footnotetext{
${ }^{15}$ Anderson y Herr (2007) proponen una serie de "criterios tentativos de validez" o de "confiabilidad" para la investigación-acción que pueden ser extensivos a los procesos de producción colectiva de saberes. Respecto de estos criterios en la documentación narrativa de experiencias pedagógicas ver Suárez (2009).
} 
desarrollo, de prácticas y conocimientos en constante revisión, de formas organizativas flexibles, que teje una comunidad pedagógica entre los sujetos e hilos que la conforman.

Además, entendemos que la experiencia que viene desarrollando la Red plantea una perspectiva integral de las prácticas de extensión, investigación y docencia en vistas a desarrollar "nuevas forma de enseñar, aprender e investigar" en contacto más directo con los actores educativos, sus problemas, demandas y necesidades. En este sentido, tanto la línea de investigación que el equipo viene desarrollando, como el trabajo en la Red y las prácticas docentes en la formación de grado se encuentran estrechamente vinculadas y son insumos permanentes que permiten enriquecer, tensionar y revisar mutuamente los desarrollos del equipo en los diversos ámbitos.

La línea de investigación que el equipo desarrolla, se focaliza en las indagaciones en torno de la configuración del saber pedagógico, la reconstrucción de la memoria escolar y las nuevas modalidades de organización colectiva de los educadores y docentes (colectivos, redes, movimientos) para la intervención político pedagógica en el campo educativo (Suárez, 2011, 2013, 2014; Suárez y Argnani, 2011). Se inscribe en una tradición de investigación educativa que advierte sobre las potencialidades teóricas y metodológicas de las nociones de "campo pedagógico" y "hegemonía” para el estudio cualitativo e interpretativo de los procesos, relaciones, sujetos e instituciones educativos, en la medida en que permiten poner el foco en las relaciones de saber y de poder que configuran los espacios, tiempos, experiencias, saberes y discursos legítimos sobre la educación, así como en las contingentes configuraciones y formaciones discursivas que en esa trama de relaciones delimitan y constituyen diferentes y desiguales posiciones de sujeto. Estos debates y desarrollos teórico-metodológicos han sido centrales no sólo para el diseño, desarrollo y revisión del trabajo de Extensión, sino que constituyeron insumos importantes para reformulaciones sucesivas del programa de la materia "Educación II Análisis sistemático de los sistemas de educación formal y no formal”"16 del Departamento de Ciencias de la Educación (FFyL-UBA). Asimismo, han sido incorporados como parte del

\footnotetext{
${ }^{16}$ El propósito de la materia es introducir a los estudiantes en la construcción y el análisis de problemas pedagógicos contemporáneos, a partir de conceptualizar a la pedagogía como un campo de saber y poder atravesado por múltiples disputas por la hegemonía. Para ello, la propuesta de enseñanza plantea un enfoque integral y complejo que pretende poner en conversación y debate conceptos y supuestos de un heterogéneo cuerpo de teorías acerca de lo social, lo cultural y lo educativo, así como incorporar a los estudiantes en diversas prácticas sistemáticas de trabajo intelectual y académico.
} 
encuadre teórico-metodológico en seminarios, créditos de investigación y créditos de práctica profesional para la Licenciatura en Ciencias de la Educación. Pero, además, el desarrollo de la propuesta de la Red en el marco de la extensión universitaria ha permitido problematizar y revisar aspectos teórico-metodológicos de los dispositivos de investigación y formación que el equipo venía implementando, permitiendo captar con mayor sutileza las tensiones, dificultades y demandas de los sujetos e instituciones participantes, así como las recreaciones y adaptaciones que los mismos realizaban al dispositivo de documentación narrativa al momento de ponerlo en marcha para el trabajo con sus colectivos docentes ${ }^{17}$.

Finalmente, nos interesa destacar que la Red, como modalidad descentrada de organización, nos puede ayudar a pensar de manera ampliada el territorio de la formación docente más allá de las definiciones, regiones o jurisdicciones oficiales en que se organiza y gobierna la educación, trazando nuevos territorios. Como señala Fernandes (2005), el territorio se constituye, al mismo tiempo, como una convención y una confrontación: dado que posee límites y fronteras, es un espacio de disputa y conflicto. Esta nueva mirada del tiempo y el espacio pedagógico desata procesos de auto-formación, de formación entre pares y de conformación de comunidad educativa. En las redes se parte de supuestos diversos, en cada una de ellas se enfatizan problemas educativos diferentes y se ensayan múltiples estrategias y acciones. Acercarnos y participar desde una propuesta de extensión universitaria a los debates teórico-metodológicos y políticopedagógicos que tienen lugar en estas instancias, busca realizar un aporte a la pedagogía respecto de "otras" formas de conocimiento y de formación de docentes.

\footnotetext{
${ }^{17}$ Estas líneas de indagación vienen siendo desarrolladas en el marco de las tesis de Maestría de las autoras de este trabajo.
} 
ANDERSON, Gary y Herr, Kathryn. El docente investigador: la investigación-acción como una forma válida de generación de conocimientos. En: Sverdlick, Ingrid (comp.), La investigación educativa. Una herramienta de conocimiento y acción. Buenos Aires: Novedades Educativas, 2007, p. 47-69.

AWAD, Miryam. Ser y hacer con otros. En: Universidad Pedagógica Nacional, Memorias. III Encuentro Iberoamericano de Colectivos Escolares y Redes de Maestros que hacen Investigación desde su Escuela. Santa Marta, Colombia, 2002.

CARDELLI, Jorge. Prólogo. En: Colectivo Argentino de Docentes que hacen Investigación desde la Escuela, Investigación educativa y trabajo en red. Debates y proyecciones. Buenos Aires: Novedades Educativas, 2009, p. 9-12.

Colectivo Argentino de Docentes que hacen Investigación desde la Escuela. Investigación educativa y trabajo en red. Debates y proyecciones. Buenos Aires: Novedades Educativas, 2009.

CONNELLY, Michael y Clandinin, Jean. Relatos de experiencia e investigación narrativa", en: Larrosa, Jorge y otros, Déjame que te cuente. Ensayos sobre narrativa y educación. Barcelona: Laertes, 1995, p. 11-60.

DUHALDE, Miguel y otros. El Colectivo y las redes de investigación como formas alternativas para la organización de la formación y el trabajo docente", En: Colectivo Argentino de Docentes que hacen Investigación desde la Escuela, Investigación educativa y trabajo en red. Debates y proyecciones. Novedades Educativas, Buenos Aires, 2009, p. 21-32.

FERNANDES, B. Mançano. Movimentos socioterritoriais e movimentos socioespaciais. Contribuçao teórica para uma leitura geográfica dos movimentos sociais. En: Revista OSAL año VI, n¹6, pp. 273- 283. Buenos Aires: CLACSO, 2005.

Red DHIE. El colectivo y las redes de investigación como formas alternativas para la organización de la formación y el trabajo docente", en: Colectivo Argentino de Docentes que hacen Investigación desde la Escuela, Investigación educativa y trabajo en red. Debates y proyecciones. Buenos Aires: Novedades Educativas, 2009, p. 21-32.

SUÁREZ, Daniel H. Espacio (auto)biográfico, investigación educativa y formación docente en Argentina: un mapa imperfecto de un territorio en expansión. En: Revista Mexicana de Investigación Educativa, vol. XIX (62) p. 763-786. Ciudad de México, 2014. Disponible en: http://www.comie.org.mx/v1/revista/visualizador.php?articulo=ART62005\&criterio=http:// www.comie.org.mx/documentos/rmie/v19/no62/pdf/62005.pdf

SUÁREZ, Daniel H. Movimientos pedagógicos de docentes en torno a los saberes escolares. Concurso para el cargo de Profesor Regular Asociado, Cátedra "Educación II - 
Análisis sistemático de los sistemas de educación formal y no formal”, Departamento de Ciencias de la Educación Facultad de Filosofía y Letras, UBA, 2013.

SUÁREZ, Daniel H. Relatos de experiencia, saber pedagógico y reconstrucción de la memoria escolar. En: Alliaud, Andrea y Suárez, Daniel H. (coords.), El saber de la experiencia: narrativa, investigación y formación docente. Buenos Aires: Facultad de Filosofía y Letras/UBA y CLACSO, 2011, p. 93-137.

\section{SUÁREZ, Daniel H. Relatos pedagógicos, docentes e investigación narrativa de la} experiencia escolar. Aportes de la investigación cualitativa y colaborativa para la formación y el desarrollo profesional de los docentes. Tesis doctoral, Facultad de Filosofía y Letras. Universidad de Buenos Aires, 2009

SUÁREZ, Daniel H. Docentes, narrativa e investigación educativa. La documentación narrativa de las prácticas docentes y la indagación pedagógica del mundo y las experiencias escolares. En: Sverdlick, Ingrid (comp.) La investigación educativa. Una herramienta de conocimiento y acción. Buenos Aires: Novedades Educativas, 2007.

SUÁREZ, Daniel H. y Argnani, Agustina. Nuevas formas de organización colectiva y producción de saber pedagógico: la Red de Formación Docente y Narrativas Pedagógicas. En: Revista Educação e Contemporaneidade, vol 20 (36), pp. 43-56. Salvador: Universidade do Estado da Bahia-UNEB/Departamento de Educação, 2011.

SUÁREZ, Daniel H., Dávila, Paula y Ochoa, Liliana. La indagación de los mundos escolares a través de la documentación narrativa de experiencias pedagógicas. Un aporte teórico y metodológico. En: Cuadernos de Educación $\mathrm{N}^{\circ}$ 7. Año VII, N ${ }^{\circ}$ 7. Centro de Investigaciones "María Saleme de Burnichón”. Facultad de Filosofía y Humanidades. Universidad Nacional de Córdoba. Córdoba, mayo de 2009.

SUÁREZ, Daniel; Dávila, Paula; Ochoa, Liliana. ¿Vale la pena documentar narrativamente experiencias pedagógicas? Una invitación. En: AAVV, Maestros contadores de historias: narrativas en red pedagógica. Medellín: Universidad de Antioquia y Escuela del Maestro, pp. 9-14, 2008

SUÁREZ, Daniel H.; Ochoa, Liliana y Dávila, Paula La documentación narrativa de experiencias pedagógicas. En: Revista Nodos y Nudos, Vol. 2; №17, p. 16-3, 2005.

UNDA BERNAL, María del Pilar y Martínez Boom, Alberto. Lugar, redes y saberes: hacia un movimiento pedagógico y cultural en Iberoamérica. En: Universidad Pedagógica Nacional, Memorias. III Encuentro Iberoamericano de Colectivos Escolares y Redes de Maestros que hacen Investigación desde su Escuela. Santa Marta, Colombia, 2002.

Universidad Pedagógica Nacional. Memorias. III Encuentro Iberoamericano de Colectivos Escolares y Redes de Maestros que hacen Investigación desde su Escuela. Santa Marta, Colombia, 2002. 
SANTOS, Boaventura de Souza. Conocer desde el Sur. Para una cultura política

emancipatoria. Lima: Fondo Editorial de la Facultad de Ciencias Sociales UNMSN, 2006.

Recebido em: 16/03/2015 Aprovado em: 30/07/2014

Universidade do Estado de Santa Catarina - UDESC Programa de Pós-Graduação em Educação - PPGE Revista Linhas Volume 16 - Número 32 - Ano 2015 revistalinhas@gmail.com 